\title{
Ellipsoidal shapes in general relativity: general definitions and an application
}

\author{
József Zsigrai* \\ Department of Physics, Hiroshima University, \\ Higashi-Hiroshima 739-8526, Japan \\ and \\ Institute for Isotope- and Surface Chemistry of the Hungarian Academy of Science \\ H-1525 Budapest, P.O. Box 77, Hungary \\ February 7, 2008
}

\begin{abstract}
A generalization of the notion of ellipsoids to curved Riemannian spaces is given and the possibility to use it in describing the shapes of rotating bodies in general relativity is examined. As an illustrative example, stationary, axisymmetric perfect-fluid spacetimes with a so-called confocal inside ellipsoidal symmetry are investigated in detail under the assumption that the 4 -velocity of the fluid is parallel to a time-like Killing vector field. A class of perfect-fluid metrics representing interior NUT-spacetimes is obtained along with a vacuum solution with a non-zero cosmological constant.
\end{abstract}

PACS numbers: 04.20.Jb, 04.40.Nr

\section{Introduction}

In the past much effort has been made to find an exact solution of Einstein's field equations representing the gravitational field of a compact rapidly rotating object, but without much success. In particular, a great deal of work has been done concerning stationary, axially symmetric perfect-fluid configurations as possible candidates for modelling the interior of rapidly rotating bodies. In addition, in

*E-mail: zsigrai@sunserv.kfki.hu 
order to give a global space-time model completely describing the gravitational field of the source, the possibilities for matching an interior perfect-fluid solution to an exterior vacuum metric have been extensively studied. So far, however, the only known physically acceptable global exact solution in the examined category is the so-called Neugebauer-Meinel disk of dust [1], which may be considered as the limiting case of uniformly rotating relativistic objects.

Apart from the above mentioned disk, in many cases encountered in the search for physically acceptable solutions it is not possible to give an obvious geometrical interpretation for the discovered configurations. On the other hand, it is well known that in Newtonian gravity numerous results have been obtained for the case in which a rotating fluid in equilibrium has an ellipsoidal shape 2]. In the present work we will constrain our attention to those rapidly rotating configurations in general relativity, for which there is a natural way for imposing the restriction that the surface of the considered object is ellipsoidal. Using the approach presented in this paper the matching surface between the interior and exterior region may be conveniently chosen to be the ellipsoid representing the boundary of the rotating fluid body. It should be emphasized, however, that other shapes for a rotating fluid might also be possible in both general relativity and Newtonian theory as well.

In the Euclidean geometry of flat space the notion of an ellipsoid is clear and easily understood. In curved spaces, however, it does not have such an obvious intuitive meaning. Therefore, in the general-relativistic analysis of perfect-fluid bodies having an ellipsoidal shape, it is necessary to determine first what an ellipsoid in curved space is. Originally, it has been proposed by Krasiński [3] to consider a 3-dimensional, axially symmetric Riemannian space in which the line element ${ }^{(3)} d s^{2}$ of the 3 -dimensional space can be written as

$$
{ }^{(3)} d s^{2}=z(\rho, \theta) d \rho^{2}+\left(\rho^{2}+a^{2} \cos ^{2} \theta\right) d \theta^{2}+\left(\rho^{2}+a^{2}\right) \sin ^{2} \theta d \phi^{2},
$$

where $a$ is a constant and $z$ is a positive function. In such spaces the coordinate surfaces $\rho=$ const represent the analogue of a one-parameter congruence of confocal Euclidean ellipsoids of revolution. Later a more general, coordinate-free definition of (not necessarily axially symmetric) Riemannian spaces that can be filled in with a one-parameter family of confocal ellipsoids was given by Rácz [4, recovering the metric (1.1) as a special case. In [3] it was assumed that the union of local rest spaces of the observers co-moving with matter has the metric (1.1), whereas in [4] it was the 3-space of timelike Killing orbits which had this ellipsoidal symmetry. By using the approach of [4], a uniqueness theorem for stationary axisymmetric vacuum spacetimes with a particular type of ellipsoidal symmetry was proven in [5]. We remark here that ellipsoidal shapes in curved spaces, but in a different context, have been considered in [6] as well (see also 
references therein).

The purpose of the present paper is to construct a framework for considering ellipsoidal shapes in general relativity, which covers the situations studied in [3] and 4 as special cases. In this way a firm mathematical basis for the ideas pioneered by Krasiński [3] is given and the constructions introduced by Rácz [4] are extended in order to include a wider class of spaces in the analysis. This work also aims to examine the applicability of the constructed framework on the particular example of stationary, axially symmetric rigidly rotating perfect fluid spacetimes with certain ellipsoidal symmetries as test models for the interior solution. It has been shown by Geroch and Lindblom [7] that rigidly rotating perfect fluids can be considered to be the equilibrium configurations of relativistic dissipative fluids so they are of obvious astrophysical interest. Although the test models considered in this paper did not provide perfect-fluid solutions with physically suitable properties, the existence of such solutions within the very wide class of spacetimes with some kind of ellipsoidal symmetry cannot be excluded.

This paper is organized as follows. First the definition of a Riemannian 3-space that can be filled in with a one-parameter congruence of concentric ellipsoids is given. In such curved spaces one can introduce a notion analogous to the notion of ellipsoids in Euclidean space. Next, the possibilities for considering this type of 3 -spaces within the framework of general relativity are discussed. The definition of a "conformally ellipsoidal spacetime" as well as the more restrictive definitions of a "spacetime with co-moving ellipsoidal symmetry" and of a "spacetime with inside ellipsoidal symmetry" are presented. As an illustration of these notions, stationary, axially symmetric, rigidly-rotating perfect-fluid configurations with "confocal" inside ellipsoidal symmetry are considered. After reviewing some basic notions pertaining to rigidly rotating fluids, our considerations are first specialized to the subcase in which the gradient of the norm of the time-like Killing vector field and its twist are linearly dependent. Making use of the integrability conditions of the field equations, the general form of the metric for the selected perfect-fluid configurations is determined and it is demonstrated that the resulting metric can be joined smoothly to the Taub-NUT solution. Some previously known exact solutions are shown to belong to the class of spacetimes studied here. In the complementary case when the norm of the gradient and the twist of the Killing field are linearly independent it is shown that a particular (possibly new) perfect-fluid solution satisfies the integrability conditions. The resulting particular metric, however, can be re-interpreted as a vacuum solution with a non-zero cosmological constant. Finally, the Appendix contains a review of some facts concerning ellipsoids in flat Euclidean space. 


\section{2 "Ellipsoids" in curved space}

In the following a possible approach for considering ellipsoidal shapes in curved Riemannian spaces is presented. In particular, the definition given in 4 is generalized and it is shown how a convenient coordinate system can be chosen in the Riemannian spaces under consideration.

Definition 1: A Riemannian 3-space $\left(\mathcal{S}, h_{a b}\right)$ can be filled in with a one-parameter congruence of concentric ellipsoids if it satisfies the following properties: (a) there exists in it a congruence of surfaces, $\mathcal{E}_{\rho}$, with induced metric $\chi_{i j}[\rho]$, (b) there exists a diffeomorphism, $\Psi$, mapping $\mathcal{S}$ into a subset, $\Psi[\mathcal{S}]$, of $\mathbf{R}^{\mathbf{3}}$ so that the tensor field $\Psi^{*} \chi_{i j}[\rho]$ is just the induced metric field for some one-parameter congruence of concentric ellipsoids in $\Psi[\mathcal{S}] \subset \mathbf{R}^{\mathbf{3}}$ and (c) for any value of $\rho$, the extrinsic curvature tensor field, $\kappa_{i j}[\rho]$, of the surfaces $\mathcal{E}_{\rho}$, embedded in $\mathcal{S}$, is conformal to the metric tensor of the Euclidean unit sphere, $\gamma_{i j}$.

We will refer to the surfaces $\mathcal{E}_{\rho}$ as "ellipsoids in curved space". Note that this definition has been obtained by generalizing the properties of Euclidean ellipsoids (given in the Appendix) to curved spaces, just as it has been done in [4. However, an essential difference between the above definition and the one given in [4] is that the term "confocal" used in [4] is replaced here by the term "concentric". This allows one to consider Riemannian spaces of a much more general character than it was the case in 4 .

By analogy with the $3+1$ decomposition of a spacetime $\left(\mathcal{M}, g_{a b}\right)$ (see, e.g., $\S 21.4$ of [8] ), a 3-dimensional Riemannian space $\left(\mathcal{S}, h_{a b}\right)$ may be decomposed in a similar way in $2+1$ dimensions. Let $\mathcal{E}_{\rho}$ denote a surface labelled by the parameter value " $\rho$ " in a one-parameter congruence of 2 -dimensional surfaces embedded in a 3-dimensional Riemannian space. Since there is always only one surface $\mathcal{E}_{\rho}$ of the congruence passing through any given point of $\mathcal{S}$, the parameter $\rho$ used for labelling the surfaces may be chosen as one of the coordinates of $\mathcal{S}$. In such a coordinate system the distance between two nearby points belonging to two different surfaces $\mathcal{E}_{\rho}$ and $\mathcal{E}_{\rho+d \rho}$ can be given as

$$
d s^{2}=\alpha^{2} d \rho^{2}+\chi_{i j}[\rho]\left(d x^{i}+\beta^{i} d \rho\right)\left(d x^{j}+\beta^{j} d \rho\right)
$$

where $\alpha$ and $\beta^{i}$ are the $2+1$ analogues of the "lapse function" and "shift vector", $\chi_{i j}[\rho]$ is the induced metric on $\mathcal{E}_{\rho}$, while $x^{i}(i, j=2,3)$ is a coordinate system on this surface. Expression (2.1) gives, in fact, the line element of the 3-dimensional Riemannian space $\left(\mathcal{S}, h_{a b}\right)$. The components of the metric $h_{a b}$ and its inverse, $h^{a b}$, are given by

$$
\left(\begin{array}{cc}
h_{11} & h_{1 j} \\
h_{i 1} & h_{i j}
\end{array}\right)=\left(\begin{array}{cc}
\alpha^{2}+\beta_{k} \beta^{k} & \beta_{j} \\
\beta_{i} & \chi_{i j}
\end{array}\right),
$$




$$
\left(\begin{array}{cc}
h^{11} & h^{1 j} \\
h^{i 1} & h^{i j}
\end{array}\right)=\left(\begin{array}{cc}
1 / \alpha^{2} & -\beta^{j} / \alpha^{2} \\
-\beta^{i} / \alpha^{2} & \chi^{i j}+\beta^{i} \beta^{j} / \alpha^{2}
\end{array}\right)
$$

The requirement that $\Psi^{*} \chi_{i j}[\rho]$ is the induced metric field for some one-parameter congruence of concentric ellipsoids in $\Psi[\mathcal{S}] \subset \mathbf{R}^{\mathbf{3}}$ implies that there exists a coordinate system $\left(x^{2}=\theta, x^{3}=\phi\right)$ in which the line element of the induced metric on the surface $\mathcal{E}_{\rho}$ can be written as (compare with equation (A.2) of the Appendix)

$$
\begin{aligned}
d s^{2}\left(\chi_{i j}[\rho]\right) & =\left[\left(x_{0}^{2} \cos ^{2} \phi+y_{0}^{2} \sin ^{2} \phi\right) \cos ^{2} \theta+\rho^{2} \sin ^{2} \theta\right] \cdot d \theta^{2} \\
& +\left(x_{0}^{2} \sin ^{2} \phi+y_{0}^{2} \cos ^{2} \phi\right) \sin ^{2} \theta \cdot d \phi^{2} \\
& +2\left(y_{0}^{2}-x_{0}^{2}\right) \sin \theta \cos \theta \sin \phi \cos \phi \cdot d \theta d \phi
\end{aligned}
$$

where $x_{0}=x_{0}(\rho)$ and $y_{0}=y_{0}(\rho)$. On the other hand, the requirement that the extrinsic curvature tensor field $\kappa_{i j}[\rho]$ is conformal to the metric of the Euclidean unit sphere, $\gamma_{i j}$, means that there exists a smooth (at least $C^{2}$ ) everywhere positive function $k(\rho, \theta, \phi)$ so that ${ }^{1}$

$$
\kappa_{i j}=k(\rho, \theta, \phi) \gamma_{i j}
$$

In general, the extrinsic curvature, or "second fundamental form", of a surface is given by the expression [9, 10]

$$
\kappa_{i j}=\frac{\partial x^{a}}{\partial \xi^{i}} \frac{\partial x^{b}}{\partial \xi^{j}} D_{\alpha} n_{b},
$$

where $n_{a}$ is the unit normal to the surface, $D_{a}$ is the covariant derivative associated with the metric of the Riemannian 3-space, $x^{a}$ are coordinates on the 3 -space, while $\xi^{i}$ are coordinates on the 2 -dimensional surface $(a, b=1,2,3$ and $i, j=2,3)$. If the surface is given by an equation of the form

$$
f\left(x^{a}\left(\xi^{i}\right)\right)=0
$$

then the unit normal can be given as

$$
n_{a}= \pm\left|h^{b c} \frac{\partial f}{\partial x^{b}} \frac{\partial f}{\partial x^{c}}\right|^{-1 / 2} \frac{\partial f}{\partial x^{a}}
$$

with the ambiguity in sign arising from the ambiguity in the orientation of the normal. In the present case $x^{1}=: \rho, x^{2}=\xi^{2}=: \theta x^{3}=\xi^{3}=: \phi$, the surface

\footnotetext{
${ }^{1}$ From now on we omit the labels " $[\rho]$ " in the notation and denote any tensor field $\tau_{i j}[\rho]$ simply as $\tau_{i j}$. Thus, the dependence on $\rho$, as well as on the coordinates $\theta$ and $\phi$, is understood.
} 
equation is given by $f=\rho-$ const $=0$, and, thus, the components of the unit normal are $\left(n_{1}, n_{2}, n_{3}\right)=( \pm \alpha, 0,0)$.

In this setup, with the aid of (2.2) and (2.3) one obtains from (2.6)

$$
\kappa_{i j}= \pm \frac{1}{2 \alpha}\left(\frac{\partial \beta_{j}}{\partial x^{i}}+\frac{\partial \beta_{i}}{\partial x^{j}}-\frac{\partial \chi_{i j}}{\partial \rho}-2 \beta_{k} \tilde{\Gamma}_{i j}^{k}\right)
$$

for the extrinsic curvature (see also [8]), where $\tilde{\Gamma}_{i j}^{k}$ are the Christoffel symbols for the metric $\chi_{i j}$, which is given by (2.4). Furthermore, in the coordinate system of (2.4), the line element of the unit sphere may be written as

$$
d s^{2}\left(\gamma_{i j}\right)=d \theta^{2}+\sin ^{2} \theta d \phi^{2}
$$

so it follows from equation (2.5) that

$$
\frac{\kappa_{(\phi \phi)}}{\kappa_{(\theta \theta)}}=\sin ^{2} \theta, \kappa_{(\theta \phi)}=0
$$

where $\kappa_{(\theta \theta)}, \kappa_{(\theta \phi)}$ and $\kappa_{(\phi \phi)}$ each denote the corresponding component of the extrinsic curvature. Since for the spaces under consideration the metric $\chi_{i j}$ has been already specified by equation (2.4), it follows from (2.9) that the system (2.11) contains as unknown functions only the two components of $\beta_{i}$.

Now it is straightforward to show that (2.11) is identically satisfied if one choses the components $\beta_{(\theta)}$ and $\beta_{(\phi)}$ of $\beta_{i}$ as

$$
\begin{aligned}
& \beta_{(\theta)}=\left[\left(x_{0} x_{0}^{\prime} \cos ^{2} \phi+y_{0} y_{0}^{\prime} \sin ^{2} \phi-\rho\right) \cos \theta+F(\rho)\right] \sin \theta, \\
& \beta_{(\phi)}=\left[\left(-x_{0} x_{0}^{\prime}+y_{0} y_{0}^{\prime}\right) \sin \phi \cos \phi+G(\rho)\right] \sin ^{2} \theta,
\end{aligned}
$$

where $F(\rho)$ and $G(\rho)$ are arbitrary functions and the prime denotes derivation with respect to $\rho$. Note that $\beta_{(\theta)}$ and $\beta_{(\phi)}$ satisfy the system (2.11) for arbitrary choice of the functions $F$ and $G$, including the particular case $F=G=0$. In fact, fixing the functions $F$ and $G$ merely corresponds to a definite choice of coordinate system. Therefore, without loss of generality, hereafter these two functions will be set to be identically zero. Correspondingly, there exists a coordinate system in which the line element of a curved Riemannian 3-space that can be filled in with a one-parameter congruence of concentric ellipsoids takes the form

$$
\begin{aligned}
d s^{2} & =z(\rho, \theta, \phi) \cdot d \rho^{2}+\left[\left(x_{0}^{2} \cos ^{2} \phi+y_{0}^{2} \sin ^{2} \phi\right) \cos ^{2} \theta+\rho^{2} \sin ^{2} \theta\right] \cdot d \theta^{2} \\
& +\left(x_{0}^{2} \sin ^{2} \phi+y_{0}^{2} \cos ^{2} \phi\right) \sin ^{2} \theta \cdot d \phi^{2} \\
& +2\left(x_{0} x_{0}^{\prime} \cos ^{2} \phi+y_{0} y_{0}^{\prime} \sin ^{2} \phi-\rho\right) \cos \theta \sin \theta \cdot d \rho d \theta \\
& +2\left(-x_{0} x_{0}^{\prime}+y_{0} y_{0}^{\prime}\right) \cos \phi \sin \phi \sin ^{2} \theta \cdot d \rho d \phi \\
& +2\left(y_{0}^{2}-x_{0}^{2}\right) \cos \phi \sin \phi \cos \theta \sin \theta \cdot d \theta d \phi
\end{aligned}
$$


where $z(\rho, \theta, \phi)$ is a $C^{2}$ function, $x_{0}$ and $y_{0}$ are functions depending merely on the coordinate ' $\rho$ ', and the prime denotes derivation. In these coordinates the $\rho=$ const surfaces are the curved-space analogues of Euclidean ellipsoids. Note also that the particular choice $z=\left(\left(x_{0}^{\prime}\right)^{2} \cos ^{2} \phi+\left(y_{0}^{\prime}\right)^{2} \sin ^{2} \phi\right) \sin ^{2} \theta+\cos ^{2} \theta$ recovers the metric of flat Euclidean space in ellipsoidal coordinates (compare with equation (A.2) of the Appendix).

The freedom carried by the functions $x_{0}$ and $y_{0}$ as well as the $\phi$-dependence of $z$ in the above form of the metric implies that (2.14) describes a much wider class of spaces than the metric (1.1). Putting, e.g., $x_{0}^{2}=\rho^{2}+a^{2}, y_{0}^{2}=\rho^{2}+b^{2}$ into (2.14) where $a$ and $b$ are constants, recovers the line element of non-symmetric Riemannian spaces that can be filled in with a congruence of confocal ellipsoids, originally given in $4^{2}$. The present paper, however, will be particularly concerned with the special case in which $z(\rho, \theta, \phi)=z(\rho, \theta)$ and $x_{0}^{2}=y_{0}^{2}=\rho^{2}+a^{2}$, where $a=$ const. Then the metric of the considered 3-dimensional Riemannian space reduces exactly to the metric (1.1). In this special case the congruence of the $\rho=$ const surfaces is the analogue of a congruence of rotationally invariant confocal oblate ellipsoids in Euclidean space.

\section{$3 \quad$ Ellipsoidal shapes in general relativity}

In the previous section we have been concerned merely with structures in 3dimensional Riemannian geometry, without connecting them to any structures in general relativity. In this section Riemannian spaces within the 4-dimensional spacetime of general relativity will be considered, assuming that these Riemannian spaces can be filled in with a one-parameter family of concentric ellipsoids, in the sense of Definition 1 from the previous section.

In general relativity a description of any shape depends on the motion of the observers performing the description, as it has been already emphasized in [3]. E.g., a body that has an ellipsoidal shape as seen by one class of observers might appear to have a completely different shape as seen by some other observers. Therefore when considering ellipsoidal shapes in general relativity, one should also specify a choice of preferred observers. To start off, consider the following construction:

Definition 2: A spacetime $\left(M, g_{a b}\right)$ is conformally ellipsoidal if there exists in it a congruence of preferred observers for which the union of their local rest

\footnotetext{
${ }^{2}$ Note, however, that the non-diagonal term with " $d \theta d \phi$ " is missing form the line element given by equation (11) in [4. Nevertheless, this omission does not influence the remaining results in [4], because they correspond to the rotationally invariant case, in which the mentioned non-diagonal term identically vanishes.
} 
spaces is conformal to a Riemannian 3 -space $\left(\mathcal{S}, h_{a b}\right)$ that can be filled in with a one-parameter congruence of concentric ellipsoids.

For the observers moving with 4 -velocity $u^{a}$, Definition 2 implies

$$
g_{a b}+u_{a} u_{b}=\Omega^{2} h_{a b},
$$

where $\Omega^{2}$ is a conformal factor. A spacetime is ellipsoidal if $\Omega^{2}=1$. Note that the original term "ellipsoidal spacetime" first introduced in [3] is equivalent to the notion of a stationary, axisymmetric spacetime with "co-moving ellipsoidal symmetry" (see Definition 3) in the terminology of the present work.

Notice now that the choice of preferred observers has still not been uniquely specified. Therefore, in accordance with the remark above Definition 2, in order to consider ellipsoidal shapes within general relativity, Definition 2 should be supplemented by a prescription for choosing the preferred observers. There is an enormous freedom in fixing the congruence of observers and the conformal factor referred to in Definition 2. In particular, we will consider two possible situations, which give rise to the definitions presented bellow, generalizing the constructions given in [3] and [4].

Let us recall that the main motivation for considering the analogues of ellipsoidal surfaces in curved spaces was examining rapidly rotating bodies having an ellipsoidal surface in the framework of general relativity. As discussed in [3], a natural 3-dimensional Riemannian space in which one should consider the ellipsoidal shapes of rotating bodies in general relativity is the union of local rest spaces of observers co-moving with matter. This serves as a motivation for Definition 3.

Definition 3: A non-empty spacetime possesses co-moving ellipsoidal symmetry if it is ellipsoidal (i.e., conformally ellipsoidal with $\Omega^{2}=1$ ) and if the class of preferred observers is exactly the class of observers co-moving with matter. Furthermore, any ellipsoidal vacuum spacetime will be said to possess co-moving ellipsoidal symmetry, by definition.

In an empty spacetime, in general, there is no way to specify a class of distinguished observers. However, if there is a Killing vector field present on spacetime, one may give, for instance, the following definition, regardless whether the spacetime is empty or non-empty:

Definition 4: A spacetime with a timelike Killing vector field $\xi^{a}$ possesses inside ellipsoidal symmetry if it is conformally ellipsoidal with $\Omega^{2}=\left(-\xi_{a} \xi^{a}\right)^{-1}=$ : $(-v)^{-1}$ and if the 4 -velocity of the preferred observers, $u^{a}$, is a unit vector field aligned with the Killing vector field, i.e., if $u^{a}=(-v)^{-1 / 2} \xi^{a}$.

In particular, if the rest spaces of the preferred observers from Definition 4 can be filled in with a congruence of confocal ellipsoids, we will refer to the spacetime 
as possessing confocal inside ellipsoidal symmetry ${ }^{3}$.

A spacetime with co-moving ellipsoidal symmetry need not, but might possess inside ellipsoidal symmetry and vice versa. For instance, the Kerr spacetime is an example of a spacetime which possesses both these types of ellipsoidal symmetry at the same time. The present paper will be mainly concerned with the case of inside ellipsoidal symmetry, while spacetimes with co-moving ellipsoidal symmetry are the subject of ongoing research. It turns out, however, that a class of metrics with inside ellipsoidal symmetry discussed in this paper possesses comoving ellipsoidal symmetry as well (see equations (4.20) and (4.21) later on in this paper).

In [5] stationary, axially symmetric empty spacetimes with confocal inside ellipsoidal symmetry have been considered ${ }^{4}$. By examining the integrability conditions of the field equations it has been demonstrated that the full set of solutions to the vacuum Einstein equations satisfying these conditions coincides with the family of the Kerr-NUT solutions with vanishing electric and magnetic charges. In the remaining part of the present paper a method similar to that of [5] will be used to study stationary, axisymmetric perfect-fluid configurations possessing confocal inside ellipsoidal symmetry and having the 4-velocity of the fluid parallel to a timelike Killing vector field.

\section{Rigidly rotating perfect-fluid spacetimes with confocal inside ellipsoidal symmetry}

\subsection{The field equations for rigidly rotating perfect fluids}

In the following we will restrict our attention to stationary, axisymmetric perfectfluid spacetimes with the energy momentum tensor

$$
T_{a b}=(\mu+P) u_{a} u_{b}+P g_{a b},
$$

where $\mu$ and $P$ are the density and pressure of the fluid and for which the 4velocity of the fluid is a unit vector field $u^{a}$ aligned with a timelike Killing field $\xi^{a}$, so that

$$
u^{a}=(-v)^{-1 / 2} \xi^{a},
$$

where $v=\xi_{a} \xi^{a}$ is the norm of the time-like Killing vector field $\xi^{a}$. Such fluids are "rigid" in the sense that they are expansion- and shear-free. Moreover, for the

\footnotetext{
${ }^{3}$ We remark that the term "inside ellipsoidal symmetry" originally introduced in [4] is equivalent to the term "confocal inside ellipsoidal symmetry" used in the present work.

${ }^{4}$ Note that [5] refers to "ellipsoidal spacetimes" which are, in fact, spacetimes with confocal inside ellipsoidal symmetry in the terminology of the present paper.
} 
selected configurations the twist of the Killing field, defined by $\omega_{a}:=\varepsilon_{a b c d} \xi^{b} \nabla^{c} \xi^{d}$, can be given as a gradient of a function, that is, $\omega_{a}=\nabla_{a} \omega$, where $\omega$ is called the twist potential. The existence of the Killing field allows one to use the projection formalism of general relativity (for a review see, e.g., [11, 12, 13, 14]) and to write the field equations for the selected perfect-fluid configurations in the form [15, 16.

$$
\begin{gathered}
R_{a b}-16 \pi v^{-1} P h_{a b}=\frac{1}{2} v^{-2}\left[\left(D_{a} v\right)\left(D_{b} v\right)+\left(D_{a} \omega\right)\left(D_{b} \omega\right)\right], \\
D_{m} D^{m} v=v^{-1}\left[\left(D_{m} v\right)\left(D^{m} v\right)-\left(D_{m} \omega\right)\left(D^{m} \omega\right)\right]-8 \pi(\mu+3 P), \\
D_{m} D^{m} \omega=2 v^{-1}\left(D_{m} \omega\right)\left(D^{m} v\right),
\end{gathered}
$$

where $R_{a b}$ and $D_{a}$ are the Ricci-tensor and covariant derivative associated with the 3-dimensional Riemannian metric $h_{a b}$ on the space of time-like Killing orbits defined by

$$
(-v)^{-1} h_{a b}=g_{a b}-\frac{1}{v} \xi_{a} \xi_{b} .
$$

In addition to the above equations, the equation of motion

$$
\partial_{a} P+\frac{1}{2}(\mu+P) \frac{\partial_{a} v}{v}=0
$$

which follows from the above ones, is also satisfied.

In the spirit of Definition 4, for spacetimes with confocal inside ellipsoidal symmetry the 4 -velocity of the preferred observers is given by $u^{a}=(-v)^{-1 / 2} \xi^{a}$, i.e., in the present case of rigidly rotating fluids it coincides with the 4 -velocity of the fluid elements. Therefore, the preferred observers referred to in Definition 4 are now co-moving with the fluid. This, however, does not necessarily mean that the spacetime possesses co-moving ellipsoidal symmetry in the sense of Definition 3 , because that would also require $\Omega^{2}=1$ for the conformal factor $\Omega^{2}$ introduced in equation (3.1). In the present case $\Omega^{2}=(-v)^{-1}$ and $h_{a b}$ is expected to be the metric of an axially symmetric Riemannian 3-space that can be filled in with a one-parameter congruence of confocal ellipsoids. Thus, equation (3.1) is equivalent now to equation (4.6), which defines the metric on the space of timelike Killing orbits. Using (1.1) for the line element of the metric $h_{a b}$, it follows that in a coordinate system $\left(x^{0}=t, x^{1}=\rho, x^{2}=\theta, x^{3}=\phi\right)^{5}$ adapted to the Killing vector

\footnotetext{
${ }^{5}$ Note that, in general, the coordinates $x^{1}, x^{2}$ and $x^{3}$ of spacetime may depend on the coordinates $\rho, \theta$ and $\phi$ of the three-dimensional Riemannian space in a more complicated way. In the present case of rigidly rotating fluids with inside ellipsoidal symmetry, however, the simple identifications $x^{1}=\rho, x^{2}=\theta, x^{3}=\phi$ are possible.
} 
fields, the line element of a stationary, axially symmetric spacetime possessing confocal inside ellipsoidal symmetry can be given in the form

$$
\begin{aligned}
d s^{2} & =v(\rho, \theta)(d t+A(\rho, \theta) d \phi)^{2} \\
& -\frac{1}{v(\rho, \theta)}\left(z(\rho, \theta) d \rho^{2}+\left(\rho^{2}+a^{2} \cos ^{2} \theta\right) d \theta^{2}+\left(\rho^{2}+a^{2}\right) \sin ^{2} \theta d \phi^{2}\right)
\end{aligned}
$$

where $v(\rho, \theta), A(\rho, \theta)$ and $z(\rho, \theta)$ are the unknown metric functions. The metric function $A(\rho, \theta)$ is recovered from the quantities used in the projection formalism through the equations

$$
\frac{\partial A}{\partial \rho}=-\frac{\sqrt{u}}{v^{2}} \frac{\partial \omega}{\partial \theta} \sin \theta, \frac{\partial A}{\partial \theta}=-\frac{\rho^{2}+a^{2}}{v^{2} \sqrt{u}} \frac{\partial \omega}{\partial \rho} \sin \theta,
$$

where we have introduced the function

$$
u=u(\rho, \theta):=z(\rho, \theta) \frac{\rho^{2}+a^{2}}{\rho^{2}+a^{2} \cos ^{2} \theta} .
$$

In the coordinate system of (4.8), from the field equation (4.3) one has

$$
16 \pi v^{-1} P=\frac{R_{33}}{h_{33}}
$$

and therefore one can write

$$
H_{A B}:=R_{A B}-\frac{R_{33}}{h_{33}} h_{A B}=\frac{1}{2} v^{-2}\left[\left(\partial_{A} v\right)\left(\partial_{B} v\right)+\left(\partial_{A} \omega\right)\left(\partial_{B} \omega\right)\right],
$$

where $A, B=1,2$. Notice that the left-hand side of the above equation, i.e., the tensor $H_{A B}$ introduced here, depends merely on the 3-dimensional Riemannian metric $h_{a b}$ [15, 16. Since in the present coordinate system the only unspecified component of $h_{a b}$ is given by the function $z(\rho, \theta)$, it follows that the only unknown function entering into $H_{A B}$ is $z(\rho, \theta)$. This property will be useful in the study of the integrability conditions of the field equations, since they will reduce to a system of equations for the single unknown function $z(\rho, \theta)$ [15].

Analogously to the vacuum case considered in [5], in studying the above field equations it is convenient to consider separately two cases: one in which the twist, $D_{a} \omega$, and the gradient of the norm of the Killing field, $D_{a} v$, are linearly dependent, and the other in which they are linearly independent. 


\section{2 $\quad D_{a} v$ and $D_{a} \omega$ linearly dependent}

\subsubsection{The general form of the metric}

If $D_{a} v \neq 0$ and $D_{a} \omega \neq 0$ are linearly dependent, then the functions $v$ and $\omega$ are functionally related, so that one can write $\omega=\omega(v)$. Then using equation (4.12) one obtains

$$
H_{A B}=\frac{1}{2} \partial_{A} V \partial_{B} V
$$

where $A, B=1,2$ and we have introduced the function $V$ by

$$
\partial_{A} V:=v^{-1} \sqrt{1+(d \omega / d v)^{2}} \partial_{A} v
$$

From equation (4.13) it follows that in any local coordinate system

$$
H_{11} H_{22}-H_{12}^{2}=0
$$

Furthermore, the integrability condition for the existence of a function $V$ satisfying (4.13) can be written as

$$
H_{11} \frac{\partial H_{22}}{\partial x^{1}}-H_{12} \frac{\partial H_{11}}{\partial x^{2}}=0
$$

Equations (4.15) and (4.16) are direct generalizations of the conditions obtained for the vacuum case in [5].

Recall that $H_{A B}$ depends merely on the 3-dimensional metric $h_{a b}$, and the only unknown quantity entering $H_{a b}$ is the function $z(\rho, \theta)$. Thus, in the coordinate system $\left(x^{1}=\rho, x^{2}=\theta\right)$, the equations (4.15) and (4.16) represent a system of two partial differential equations for the single unknown function $z(\rho, \theta)$. It is straightforward to check that the choice $z=z(\rho)$ and $a=0$ is a sufficient condition for both equations (4.15) and (4.16) to be satisfied. It can be shown, however, that if the pressure of the fluid satisfies $P=P(\rho)$, then this condition is not only sufficient, but also necessary for the existence of perfect-fluid spacetimes with confocal inside ellipsoidal symmetry having $v$ and $\omega$ functionally related.

Namely, suppose that the surfaces of constant pressure are given by the coordinate ellipsoids $\rho=$ const, that is, $P=P(\rho)$. Because of the equation of motion (4.7), (if $\mu+P \neq 0$ ) this implies $v=v(\rho)$, which, in turn, implies $\omega=\omega(\rho)$, since $v$ and $\omega$ were required to be functionally related. In addition, by calculating the Ricci tensor $R_{a b}$ for the 3-dimensional metric $h_{a b}$ one obtains for the single non-diagonal component of $R_{a b}$

$$
R_{12}=-\frac{\rho}{2 u\left(\rho^{2}+a^{2}\right) \sin \theta} \frac{\partial u}{\partial \theta}
$$


where the function $u=u(\rho, \theta)$ is defined by equation (4.10). With this result, setting $a=1, b=2$ in the field equation (4.3) yields $\partial u / \partial \theta=0$, i.e., $u=u(\rho)$. Now using the metric corresponding to the examined case of spacetimes, one obtains that (4.15) reduces to

$$
a^{3}\left[\rho \frac{\partial u}{\partial \rho}-2 u(1-u)\right] \cos ^{2} \theta+a\left[\rho \frac{\partial u}{\partial \rho}+2 u(1-u)\right] \rho^{2}=0 .
$$

Since $\rho$ and $\theta$ are independent coordinates, the term in the above equation with $\cos \theta$, as well as the one without $\cos \theta$ should vanish. Excluding the solution $u=1$, which recovers Minkowski spacetime, and the trivial solution $u=0$, one obtains that for the perfect-fluid spacetimes under consideration the necessary and sufficient condition for equation (4.18) to be satisfied is $a=0$. With this condition equation (4.16) is also identically satisfied.

On the other hand, $a=0$ and $u=u(\rho)$ imply $u=z=z(\rho)$, so the system of equations (4.9) for recovering the metric function $A$ from the quantities used in the projection formalism now reduces to the single equation

$$
\frac{1}{\sin \theta} \frac{d A}{d \theta}=-v^{-2} \rho^{2} z^{-1 / 2} \frac{d \omega}{d \rho}=: 2 R,
$$

where the constant $R$ could be introduced because the left-hand side of (4.19) depends merely on the variable $\theta$, while the right-hand side depends merely on $\rho$, so both sides should be equal to a constant. Then it follows that $A=2 R \cos \theta$. Therefore, using (4.8), the line element of all stationary, axially symmetric, rigidly rotating perfect-fluid configurations with confocal inside ellipsoidal symmetry for which the gradient of the norm of the timelike Killing vector field and its twist are linearly dependent can be given as

$$
d s^{2}=v(\rho)(d t+2 R \cos \theta d \phi)^{2}-\frac{1}{v(\rho)}\left(z(\rho) d \rho^{2}+\rho^{2}\left(d \theta^{2}+\sin ^{2} \theta d \phi^{2}\right)\right)
$$

provided that the surfaces of constant pressure are given by the $\rho=$ const ellipsoids and provided that $\mu+P \neq 0$ [17. Equivalently, by performing the coordinate transformation $-v(\rho)^{-1} \rho^{2} \rightarrow \rho^{2}$ and introducing the function $f(\rho):=-z(\rho) / v(\rho)$ one may write the metric also in the form

$$
d s^{2}=v(\rho)(d t+2 R \cos \theta d \phi)^{2}+f(\rho) d \rho^{2}+\rho^{2}\left(d \theta^{2}+\sin ^{2} \theta d \phi^{2}\right),
$$

which corresponds to a special case of spacetimes with co-moving ellipsoidal symmetry in the sense of Definition 3.

The algebraic type of the metrics (4.20) and (4.21) is Petrov type D. Unfortunately, they are not suitable for describing the interior of compact rotating bodies 
with a regular axis of rotation. Namely, the existence of a regular rotation axis would require $g_{\phi \phi} \rightarrow 0$ as $\theta \rightarrow 0$, for arbitrary $\rho$. This, however, is not possible with the metrics (4.20) or (4.21), therefore they cannot have a regular rotation axis. It turns out, instead, that they are locally rotationally symmetric and they belong to Class I in the Stewart-Ellis classification [18] of locally rotationally symmetric spacetimes.

Next we show that these perfect-fluid spacetimes can be matched to an external Taub-NUT solution, provided that there exists a $P=0$ surface. The line-element of the Taub-NUT metric is

$$
\begin{gathered}
d s^{2}=-F(r)(d \tau+2 l \cos \theta d \phi)^{2}+F^{-1}(r) d r^{2}+\left(r^{2}+l^{2}\right)\left(d \theta^{2}+\sin ^{2} \theta d \phi^{2}\right), \\
F(r)=\frac{r^{2}-2 m r-l^{2}}{r^{2}+l^{2}},
\end{gathered}
$$

where $l$ and $m$ are constants [1]. For the purpose of the matching we assume that there exists a $\rho_{s}$ for which $P\left(\rho_{s}\right)=0$ and that on the $\rho=\rho_{s}$ surface the relationships $\tau=\eta t$ ( $\eta=$ const) and $r=r(\rho)$ hold. We join the two spacetimes by requiring the continuity of the metric components at the junction surface. In addition, the components of the extrinsic curvature tensor of the matching surface are also expected to be equal on the two sides of the surface. Then one can express the parameters $m$ and $l$ of the Taub-NUT metric, as well as the junction parameter $\eta$, in terms of the functions of the interior spacetime evaluated on the surface $P=0$. That is, there exists a matching of the examined perfect-fluid spacetimes to the Taub-NUT metric with

$$
\begin{gathered}
\eta^{2}=\frac{\rho_{s}^{2} v_{s} f_{s}}{R^{2} v_{s} f_{s}-\rho_{s}^{2}} \\
l=\eta R \\
m=\frac{1}{2 \sqrt{\rho_{s}^{2}-\eta^{2} R^{2}}}\left(\rho_{s}^{2}-2 \eta^{2} R^{2}+\frac{v_{s} \rho_{s}^{2}}{\alpha^{2}}\right)
\end{gathered}
$$

where $v_{s}=v\left(\rho_{s}\right)$ and $f_{s}=f\left(\rho_{s}\right)$. Note that matchings of locally rotationally symmetric spacetimes to the Taub-NUT metric were considered in [19] as well.

\subsubsection{Examples of exact solutions}

An interesting property of the metric (4.20) is that if the function $v(\rho)$ is known, then $z(\rho)$ can be obtained from equation (4.12) by performing two quadratures. Then the pressure and density of the fluid are obtained from equations (4.7) and (4.11), respectively, without performing any integrations. Therefore, generating 
new exact solutions of this type is, in fact, a simple exercise. Unfortunately, this way there is no guarantee that the resulting spacetime will possess a meaningful equation of state. Thus, instead of giving a bunch of unphysical new exact solutions, in the following we rather list some interesting examples of previously known perfect-fluid spacetimes which can be shown to belong to the class given by equations (4.20) or (4.21). We wish to emphasize, however, that the list of previously known perfect-fluid solutions possessing confocal inside ellipsoidal symmetry presented here is by no means meant to be complete, there might as well exist other solutions in the literature, also belonging to the examined class.

In [20, 21] a spacetime originally given by Ferwagner [22] and later rediscovered by Marklund [23] is extensively studied. This spacetime represents an incompressible, rigidly rotating, Petrov type $\mathrm{D}$ perfect-fluid configuration. By performing the coordinate transformation $R \sin \chi \rightarrow \rho, d t \rightarrow d t+\left(R^{2} / \rho^{2}\right)\left(1-\rho^{2} / R^{2}\right)^{-\frac{1}{2}} d \rho$ on the metric given in [20], one arrives at

$$
d s^{2}=-\frac{\rho^{4}}{R^{4}}(d t+2 R \cos \theta d \phi)^{2}+\frac{1}{1-\rho^{2} / R^{2}} d \rho^{2}+\rho^{2}\left(d \theta^{2}+\sin ^{2} \theta d \phi^{2}\right),
$$

where $R$ is a constant. The above line element has exactly the same form as (4.21), with $v(\rho)=\rho^{4} / R^{4}$ and $f(\rho)=\left(1-\rho^{2} / R^{2}\right)^{-1}$. The density and pressure of the fluid are given by

$$
8 \pi \mu=\frac{6}{R^{2}}, 8 \pi P=\frac{4}{\rho^{2}}-\frac{6}{R^{2}}
$$

Next, it has been shown in 24] that if $\omega=\omega(v)$ and $\mu+3 P \neq 0$, there exists such a coordinate system $\left(t, x^{1}, x^{2}, \phi\right)$ in which the line element of all stationary, axisymmetric, rigidly rotating perfect-fluid spacetimes can be written as

$d s^{2}=v\left(x^{1}\right)\left(d t^{2}+A\left(x^{2}\right) d \phi^{2}\right)+\frac{1}{v\left(x^{1}\right)}\left\{h_{11}\left(x^{1}\right)\left(d x^{1}\right)^{2}+h_{22}\left(x^{1}\right)\left[\left(d x^{2}\right)^{2}+e^{2}\left(x^{2}\right) d \phi^{2}\right]\right\}$,

where all possible forms of the functions $e\left(x^{2}\right)$ and $A\left(x^{2}\right)$ are listed in 24. By setting $x^{1}=\rho, x^{2}=\theta, h_{22}=\rho^{2}$ and with the allowed choice $e^{2}=\sin ^{2} \theta$, which implies $A=2 R \cos \theta$, one recovers the general form (4.20) of the studied ellipsoidal spacetimes. An exact solution of the examined type was also given in 24, which, because of its complexity, we do not list here but rather refer the reader to equations (49)-(53) in the original article [24. Finally, in [25] by using the 3-dimensional spin coefficient method the authors have arrived to a line element which has, up to a coordinate transformation, the same general form as that given by equation (4.20), but they could obtain an exact non-vacuum solution only for the dust case. 
Note that for the special case of rigidly rotating dust one has $P \equiv 0$ and $v \equiv$ const. Assuming that the metric has the general form given by (4.20), it turns out that the only rigidly rotating dust solution satisfying the conditions (4.15) and (4.16) is given by $z(\rho)=\rho^{2} /\left(\rho^{2}+v^{2} R^{2}\right)$. This solution is identical (up to a coordinate transformation and up to renaming the constants) to a dust solution given in [25].

\section{3 $D_{a} v$ and $D_{a} \omega$ linearly independent - an exact solution}

When $D_{a} v$ and $D_{a} \omega$ are linearly independent, the general form of the metric is given by (4.8). In this case the components of the tensor $H_{A B}$, defined by (4.12), may be considered as components of a non-singular Riemannian 2-metric. It has been demonstrated in [15] that the necessary and sufficient condition for the existence of the functions $v(\rho, \theta)$ and $\omega(\rho, \theta)$ satisfying (4.12) is that the Gaussian curvature of this 2-metric is exactly minus one. In the present case this condition represents a non-linear partial differential equation for the function $z(\rho, \theta)$. It can be shown that the particular choice

$$
z(\rho, \theta)=\frac{\rho^{2}+a^{2} \cos ^{2} \theta}{\rho^{2}+a^{2}} \frac{\rho^{2}}{m^{2}-\rho^{2}},
$$

where $a$ and $m$ are some constants, satisfies the mentioned integrability condition. With this choice for $z$, the metric (4.8) satisfies the full set of field equations only if the remaining metric functions are given by

$$
v(\rho, \theta)=-C^{2}\left(\rho^{2}+a^{2} \cos ^{2} \theta\right), A(\rho, \theta)=\frac{a \sin ^{2} \theta}{C^{2}\left(\rho^{2}+a^{2} \cos ^{2} \theta\right)},
$$

where $C$ is a constant. With these functions the metric of spacetime acquires the remarkably simple form

$$
\begin{aligned}
d s^{2}= & -C^{2}\left(\rho^{2}+a^{2} \cos ^{2} \theta\right) d t^{2}+2 a \sin ^{2} \theta d t d \phi+ \\
& \frac{1}{C^{2}}\left(\frac{\rho^{2} d \rho^{2}}{\left(\rho^{2}+a^{2}\right)\left(m^{2}-\rho^{2}\right)}+d \theta^{2}+\sin \theta^{2} d \phi^{2}\right) .
\end{aligned}
$$

It is interesting to note that when the Kerr metric is recast into the form (4.8) (see 4]) then the function $z(\rho, \theta)$ for the Kerr metric is given by

$$
z_{K e r r}(\rho, \theta)=\frac{\rho^{2}+a^{2} \cos ^{2} \theta}{\rho^{2}+a^{2}} \frac{\rho^{2}}{\rho^{2}-m^{2}}
$$

which differs from (4.30) merely by a negative sign factor. 
Expression (4.32) represents an exact solution of Einstein's equations corresponding to a stationary, axially symmetric, perfect-fluid spacetime with confocal inside ellipsoidal symmetry, which does not belong to the class of solutions given in the previous subsection. For $\theta \rightarrow 0$ one has $g_{\phi \phi} \rightarrow 0$ which is compatible with the existence of a regular rotation axis. From equation (4.11) one obtains the pressure of the fluid, $P$. Then, using the equation of motion (4.7), the density of the fluid, $\mu$, can also be determined. For the particular metric (4.32) it turns out that $\mu=-P=C^{2}$, i.e., the solution can be re-interpreted as a vacuum solution with a non-zero cosmological constant $\Lambda=C^{2}$. We emphasize, however, that other perfect-fluid metrics (i.e., different from (4.32)), satisfying the integrability conditions for spacetimes with $D_{a} v$ and $D_{a} \omega$ linearly independent and possessing confocal inside ellipsoidal symmetry might exist as well. Proving the existence (or non-existence) of such metrics is still an open issue.

\section{Conclusion}

In this paper a framework has been presented which can be useful in the generalrelativistic study of ellipsoidal shapes. This framework relies on the notion of a Riemannian 3-space that can be filled in with a one-parameter congruence of concentric ellipsoids, by the use of which one can define a conformally ellipsoidal spacetime. Since in general relativity the description of any shape depends on the relative motion of the observers performing the description, in order to carry out a general-relativistic study of ellipsoidal shapes one first has to specify a choice of preferred observers. Different choices give rise to the definitions of, e.g., spacetimes with co-moving ellipsoidal symmetry and spacetimes with inside ellipsoidal symmetry.

An attempt to apply the presented framework in the study of compact, rapidly rotating bodies has been made. In particular, the applicability of the framework was tested by a detailed study of stationary, axially symmetric, rigidly rotating perfect-fluid spacetimes with confocal inside ellipsoidal symmetry. In the subcase when the twist, $D_{a} \omega$, and the gradient of the norm of the time-like Killing vector field, $D_{a} v$, are linearly related the angular dependence of the metric has been determined under the assumption that the surfaces of constant pressure are given by the $\rho=$ const "ellipsoids". The obtained general form of the metric (see equations (4.21) and (4.20) ) contains two unspecified functions of the coordinate $\rho$ and it represents a Petrov type D, locally rotationally symmetric fluid configuration. Since this metric cannot have a regular axis of rotation, it should not be expected to describe the gravitational field of a compact rotating body, but rather, as it has been shown, interior NUT spacetimes. Some previously known exact solu- 
tions belonging to this class have been recalled, and it has been indicated that new solutions of this type can be easily constructed, although their physical significance is questionable. In the complementary subcase in which $D_{a} \omega$ and $D_{a} v$ are linearly independent a possibly new exact perfect-fluid solution possessing a regular axis of rotation has been obtained. Unfortunately, it has the equation of state $\mu=-P=C^{2}$ and therefore corresponds to a vacuum configuration with a non-zero cosmological constant.

The question whether in the latter subcase one might obtain perfect-fluid solutions appropriate for describing the interior of rapidly rotating compact objects with a regular rotation axis and a physically meaningful equation of state is still an open issue. Furthermore, it would be instructive to check whether the results obtained here still remain valid if one drops the assumption that the surfaces of constant pressure are given by the $\rho=$ const surfaces and assumes merely that the surface $P=0$ is given by a coordinate ellipsoid. An additional step forward along the line of research presented here would be to alter the choice of preferred observers and to consider, e.g., spacetimes with co-moving instead of inside ellipsoidal symmetry. These issues are the subject of ongoing work.

\section{Acknowledgements}

I wish to thank I. Rácz for fruitful discussions and his helpful comments on the manuscript. This research has been made possible through the post-doctoral fellowship scheme of the Japan Society for the Promotion of Science under fellowship number P00787 and it has been also supported in part by the Hungarian National Scientific Research Fund, OTKA, grant No. T030374.

\section{Appendix}

\section{A Ellipsoids in flat space}

This appendix contains a review of the properties of ellipsoids in flat Euclidean space on which Definition 1 in the main part of this paper is based. Note that the definition given in [4] also relies on the properties of Euclidean ellipsoids listed in this Appendix.

In [3] an analysis of a 3-dimensional Euclidean space filled with a congruence of ellipsoids of revolution with a common center and common axis of symmetry was given. In order to remain inside the class of axially symmetric configurations, the following problem was left out from [3] and left for further considerations: 
Problem I. Make an analysis for nonsymmetric ellipsoids, analogous to that given for symmetric ellipsoids in [3].

To consider this problem, imagine a 3 -dimensional Euclidean space, $\mathbf{R}^{\mathbf{3}}$, filled with a congruence of ellipsoids with a common center. Let one of the semiaxes of the ellipsoids be taken as the coordinate $\rho$ in space, so that $\rho=$ const on a fixed ellipsoid. Let $\phi$ be the angle measured around this axis, and let $\theta$ be a third coordinate defined at will. Analogously to the symmetric case considered in [3], we define $(\rho, \theta, \phi)$ in terms of Cartesian coordinates $(x, y, z)$ as follows:

$$
\begin{aligned}
x & =x_{0}(\rho) \sin \theta \cos \phi \\
y & =y_{0}(\rho) \sin \theta \sin \phi \\
z & =\rho \cos \theta,
\end{aligned}
$$

where $x_{0}(\rho)$ and $y_{0}(\rho)$ are arbitrary functions whose values equal the lengths of the other two semiaxes of the $\rho=$ const ellipsoid. In these coordinates one obtains the line element $d s_{E}^{2}:=d x^{2}+d y^{2}+d z^{2}$ of Euclidean space $\mathbf{R}^{\mathbf{3}}$ as:

$$
\begin{aligned}
d s_{E}^{2} & =\left[\left(\left(x_{0}^{\prime}\right)^{2} \cos ^{2} \phi+\left(y_{0}^{\prime}\right)^{2} \sin ^{2} \phi\right) \sin ^{2} \theta+\cos ^{2} \theta\right] \cdot d \rho^{2} \\
& +\left[\left(x_{0}^{2} \cos ^{2} \phi+y_{0}^{2} \sin ^{2} \phi\right) \cos ^{2} \theta+\rho^{2} \sin ^{2} \theta\right] \cdot d \theta^{2} \\
& +\left(x_{0}^{2} \sin ^{2} \phi+y_{0}^{2} \cos ^{2} \phi\right) \sin ^{2} \theta \cdot d \phi^{2} \\
& +2\left(x_{0} x_{0}^{\prime} \cos ^{2} \phi+y_{0} y_{0}^{\prime} \sin ^{2} \phi-\rho\right) \cos \theta \sin \theta \cdot d \rho d \theta \\
& +2\left(-x_{0} x_{0}^{\prime}+y_{0} y_{0}^{\prime}\right) \cos \phi \sin \phi \sin ^{2} \theta \cdot d \rho d \phi \\
& +2\left(y_{0}^{2}-x_{0}^{2}\right) \cos \phi \sin \phi \cos \theta \sin \theta \cdot d \theta d \phi
\end{aligned}
$$

where the prime denotes derivation with respect to $\rho$.

The special case in which the congruence consists of confocal (oblate) ellipsoids is recovered by setting $x_{0}^{2}=\rho^{2}+a^{2}, y_{0}^{2}=\rho^{2}+b^{2}$, where $a$ and $b$ are constants. On the other hand, for the special case of rotationally invariant ellipsoids, we have $x_{0}=y_{0}=: g$, which reproduces equation (3.2) of [3]:

$$
\begin{aligned}
d s_{E}^{2} & =\left(\left(g^{\prime}\right)^{2} \sin ^{2} \theta+\cos ^{2} \theta\right) d \rho^{2}+2\left(g g^{\prime}-\rho\right) \sin \theta \cos \theta d \rho d \theta \\
& +\left(g^{2} \cos ^{2} \theta+\rho^{2} \sin ^{2} \theta\right) d \theta^{2}+g^{2} \sin ^{2} \theta d \phi^{2}
\end{aligned}
$$

Finally, if one considers a congruence of rotationally invariant confocal oblate ellipsoids, one has $g^{2}=\rho^{2}+a^{2}$ and the metric becomes

$$
d s_{E}^{2}=\frac{\rho^{2}+a^{2} \cos ^{2} \theta}{\rho^{2}+a^{2}} d \rho^{2}+\left(\rho^{2}+a^{2} \cos ^{2} \theta\right) d \theta^{2}+\left(\rho^{2}+a^{2}\right) \sin ^{2} \theta d \phi^{2} .
$$


Note that by letting $\rho=$ const in any of the above metrics one obtains the metric induced on a single ellipsoidal surface with the "radius" $\rho$. By using the coordinate system of (A.2) it is straightforward to show that the extrinsic curvature tensor $\kappa_{a b}$ of any one of the $\rho=$ const surfaces is conformal to the metric of the unit sphere, $\gamma_{a b}$, which may be given as $d s^{2}\left(\gamma_{a b}\right)=d \theta^{2}+\sin \theta d \phi^{2}$. That is

$$
\kappa_{a b}=k \gamma_{a b},
$$

where $k$ is a conformal factor, which is a function of the coordinates chosen to represent 3-dimensional Euclidean space. On the other hand, the intrinsic geometry of a singe surface does not depend on the properties of the congruence in which it is embedded in. Namely, in Euclidean space the surface of any $\rho=$ const ellipsoid is conformal to the unit sphere, which implies that one can always introduce such coordinates on the surface in which the metric of the surface can be put into the form $K \gamma_{a b}$, where $K$ is again some conformal factor. This is an intrinsic property of each $\rho=$ const ellipsoid. In this respect, equation (A.5) provides a connection between the "intrinsic" and "extrinsic" properties of the ellipsoidal surfaces in Euclidean space.

\section{References}

[1] Neugebauer G and Meinel R 1992 Self-gravitating disks in general relativity Astron. Gesellschaft Abstract Ser. 7, 54-54; Neugebauer G and Meinel R 1993 The Einsteinian gravitational field of the rigidly rotating disk of dust ApJ 414, no. 2 L97-L99; Neugebauer G and Meinel R 1994 General Relativistic Gravitational Field of a Rigidly Rotating Disk of Dust: Axis Potential, Disk Metric, and Surface Mass Density Phys. Rev. Lett. 73 Issue 16, 2166-2168; Neugebauer G and Meinel R 1995 General Relativistic Gravitational Field of a Rigidly Rotating Disk of Dust: Solution in Terms of Ultraelliptic Functions Phys. Rev. Lett. 75 Issue 17, 3046-3047 (gr-qc/0302060)

[2] Chandrasekhar S 1987 Ellipsoidal Figures of Equilibrium (New York, Dover Publications Inc)

[3] Krasiński A 1978 Ellipsoidal Space-Times, Sources for the Kerr Metric Ann. Phys. 112, 22-40

[4] Rácz I 1992 Note on stationary-axisymmetric vacuum spacetimes with inside ellipsoidal symmetry Class. Quantum Grav. 9, L93-L98

[5] Rácz I and Süveges M 1998 On the uniqueness of stationary axisymmetric ellipsoidal vacuum configurations Mod. Phys. Lett. A 13, No. 14, 1101-1107 
[6] Vacaru S I 2001 A New Method of Constructing Black Hole Solutions in Einstein and 5D Gravity, Preprint hep-th/0110250 Vacaru S I and Singleton D 2002 Ellipsoidal, Cylindrical, Bipolar and Toroidal Wormholes in 5D Gravity, J. Math. Phys. 43 2486-2504 (hep-th/0110272); Vacaru S I 2001 Ellipsoidal Black Hole - Black Tori Systems in 4D Gravity, Preprint hep-th/0111166 Vacaru S I 2002 Horizons and Geodesics of Black Ellipsoids, Preprint gr-qc/0206014, Vacaru S I 2002 Horizons and Geodesics of Black Ellipsoids with Anholonomic Conformal Symmetries, Preprint gr-qc/0206015; Vacaru S I 2002, Perturbations and Stability of Black Ellipsoids, Preprint gr-qc/0206016, Vacaru S I and Dehnen H 2003 Locally Anisotropic Structures and Nonlinear Connections in Einstein and Gauge Gravity Gen. Rel. Grav. 35 209-250 (gr-qc/0009039)

[7] Geroch R and Lindblom L 1991 Causal Theories of Dissipative Relativistic Fluids Ann. Phys. 207 394-416

[8] Misner C, Thorne K and Wheeler J A 1973 Gravitation (San Francisco, W.H. Freeman and Co.)

[9] Dyer C C and Oliwa C 2001 Matching of Friedman-Lemaitre-RobertsonWalker and Kasner cosmologies Class. Quantum Grav. 18, 2719-2729

[10] Musgrave P and Lake K 1996 Junctions and thin shells in general relativity using computer algebra: I. The Darmois-Israel formalism Class. Quantum Grav. 13 1885-1899 (gr-qc/9510052, 1995)

[11] Kramer D, Stephani H, MacCallum M and Herlt E 1980 Exact solutions of Einstein's field equations (Cambridge, Cambridge University Press)

[12] Geroch R 1971 A Method for Generating Solutions of Einstein's Equations J. Math. Phys. 12, No. 6, 918-924

[13] Zsigrai J 2000 A Generalized Method for Constructing Perfect-Fluid Solutions of Einstein's Equations Prog. Theor. Phys. 104, No. 2, 359-384

[14] Maison D 2000 in Einstein's Field Equations and Their Physical Implications, ed. by Schmidt B G Lect. Notes Phys. 540 273-324

[15] Rácz I 1997 On Einstein's equations for spacetimes admitting a non-null Killing field J. Math. Phys. 38, No. 8, 4237-4254 (gr-qc/9311006, 1993)

[16] Rácz I 1995 On Einstein's Equations for Perfect Fluid Sources Possessing 4velocity Parallel to a Timelike Killing Field, in Inhomogeneous Cosmological 
Models, eds. Molina A and Senovilla J M M, 222-226 (Singapore, World Scientific)

[17] Zsigrai J 2002 A Class of Rigidly Rotating Ellipsoidal Perfect-Fluid Spacetimes, in Proceedings of the Eleventh Workshop on General Relativity and Gravitation, eds. Koga J, Maeda K, Nakamura T and Tomita K, 191-195, (Tokyo, Waseda University)

[18] Stewart J M and Ellis G F R 1968 Solutions of Einstein's Equations for a Fluid Which Exhibit Local Rotational Symmetry J. Math. Phys. 9, No. 7, 1072-1082

[19] Bradley M, Fodor Gy, Gergely L Á, Marklund M and Perjés Z 1999 Rotating perfect fluid sources of the NUT metric Class. Quantum Grav. 16, 1667-1675 (gr-qc/9807058, 1998)

[20] Gergely L Á, Perjés Z and Fodor Gy 1998 Rotating Incompressible Perfect Fluid Source of the NUT Metric, Preprint gr-qc/9811056

[21] Perjés Z, Fodor Gy, Gergely L Á and Marklund M 1998 A Rotating Incompressible Perfect Fluid Space-time, Preprint gr-qc/9806095

[22] Ferwagner R 1992 Solutions of Einstein's field equations for a rigidly rotating perfect fluid, in Relativity Today, ed. Perjés Z, (Nova Science Publishers, Inc) 133, as cited in [21]

[23] Marklund M 1997 Invariant construction of solutions to Einstein's field equations - LRS perfect fluids I Class. Quantum Grav. 14, 1267-1284 (gr-qc/9612014, 1996)

[24] Herlt E 1988 Stationary Axisymmetric Perfect Fluid Solutions in General Relativity Gen. Rel. Grav. 20, No. 7, 635-646

[25] Lukács B, Newman E T, Sparling G and Winicour J 1983 A NUT-Like Solution with Fluid Matter Gen. Rel. Grav. 15, 567-579 